Article

\title{
Smenospongine, a Sesquiterpene Aminoquinone from a Marine Sponge, Induces G1 Arrest or Apoptosis in Different Leukemia Cells
}

Dexin Kong ${ }^{1, \#}$, Shunji Aoki ${ }^{1}$, Yoshihiro Sowa ${ }^{2}$, Toshiyuki Sakai ${ }^{2}$ and Motomasa Kobayashi ${ }^{1, *}$

1 Graduate School of Pharmaceutical Sciences, Osaka University, Yamada-oka 1-6, Suita, Osaka 565-0871, Japan

2 Department of Preventive Medicine, Kyoto Prefectural University of Medicine, KawaramachiHirokoji, Kamigyo-ku, Kyoto 602-8566, Japan

\# Present address: Division of Molecular Pharmacology, Cancer Chemotherapy Center, Japanese Foundation for Cancer Research, Tokyo 135-8550, Japan

* Author to whom correspondence should be addressed. Email: kobayasi@phs.osaka-u.ac.jp

Received: 23 January 2008; in revised form: 10 July 2008 / Accepted: 14 July 2008 / Published: 26 August 2008

\begin{abstract}
Smenospongine, a sesquiterpene aminoquinone isolated from the marine sponge Dactylospongia elegans, was previously reported by us to induce erythroid differentiation and G1 phase arrest of K562 chronic myelogenous leukemia cells. In this study, we investigated the effect of smenospongine on the cell cycles of other leukemia cells, including HL60 human acute promyelocytic leukemia cells and U937 human histiocytic lymphoma cells by flow cytometric analysis. Smenospongine induced apoptosis dosedependently in HL60 and U937 cells. The smenospongine treatment increased expression of p21 and inhibited phosphorylation of Rb in K562 cells, suggesting the p21-Rb pathway play an important role in G1 arrest in K562 cells. However, the p21 promoter was not activated by the smenospongine treatment based on a luciferase assay using the transfected K562 cells. Smenospongine might induce p21 expression via another mechanism than transactivation of $\mathrm{p} 21$ promoter.
\end{abstract}

Keywords: Smenospongine; G1 arrest; apoptosis; leukemia cells; p21; Rb 


\section{Introduction}

Chronic myelogenous leukemia (CML) is a hematopoietic stem cell cancer caused by the Bcr-Abl tyrosine kinase which arised from Philadelphia chromosome $(\mathrm{Ph})$ translocation [1,2]. The only known curative therapy for CML is allogeneic bone marrow transplant (BMT). However, the treatmentrelated toxicity is severe, with reported mortality at about 30\% [3]. A selective inhibitor of Bcr-Abl, imatinib mesylate $\left(\right.$ Glivec $\left.^{\circledR}\right)$, was developed as the first molecule-targeted anticancer drug [4]. Within the last decade, imatinib has proven to be highly efficacious for treatment of CML. However, with the widespread use of this drug, the resistance in some patients has been well reported, which is usually caused by mutations within Bcr-Abl $[3,5,6]$. Therefore, development of alternative drugs to imatinib for CML treatment has been expected. In addition, since Bcr-Abl is only present in CML and about $20 \%$ of cases with acute lymphoblastic leukemia, imatinib shows its limitations on other leukemia diseases. On the contrary, it was reported that FK228, a histone deacetylase (HDAC) inhibitor, was indicated to be therapeutically effective in treating not only CML, but also AML (acute myelogenous leukemia) and lymphocytic leukemia [7-9]. Consequently, there is great interest in discovering and developing similar antiumor agents showing therapeutic effects on all leukemias.

As a part of our discovery of new anticancer drug candidate from natural resources, we have been searching for new differentiation-inducing substances or cell cycle inhibitors for CML cells [10,11]. With the guidance of a bioassay method for erythroid differentiation of K562 CML cells, we isolated smenospongine, a sesquiterpene aminoquinone, from the Indonesian marine sponge Dactylospongia elegans. Smenospongine induced cell cycle arrest at the G1 phase and increased p21 expression $[12,13]$. One question, whether smenospongine shows the same effect on other leukemia cells or not, remained unclear. We recently investigated the effect of smenospongine on other leukemia cells such as HL60 and U937 by cell cycle analysis and further investigated the mechanism for the G1 arrest in K562 cells.

\section{Results and Discussion}

\subsection{Effect of smenospongine on cell cycle distribution in K562, HL60, and U937 cells}

Our previous study showed that smenospongine (Figure 1) induced G1 arrest in K562 cells [12]. To investigate whether this compound has the same effect on other leukemia cells or not, cell cycle analysis for HL60 and U937 cells together with K562 cells was carried out. Various concentrations of smenospongine as DMSO solution were added to the randomly cultured K562, HL60, and U937 cells, respectively. After $24 \mathrm{~h}$, the cells were collected for cell cycle analysis. As a result, smenospongine induced G1 phase arrest in K562 cells at $15 \mu \mathrm{M}$ concentration (Figure 2A), which is consistent with our previous report [12]. G1 arrest was not found in HL60 and U937 cells, but sub-G1 accumulation, which indicates apoptosis, was observed. And the induction of apoptosis was shown to be dosedependently (Figure 2B, 2C). In order to check whether this is attributed to the difference of the sensitivity for individual cells or not, we further analyzed with lower doses of smenospongine which did not induce apoptosis in both cells. However, no G1 accumulation was detected yet in both cells (data not shown). This suggests that smenospongine might affect different leukemia cells via the respective mechanism. 


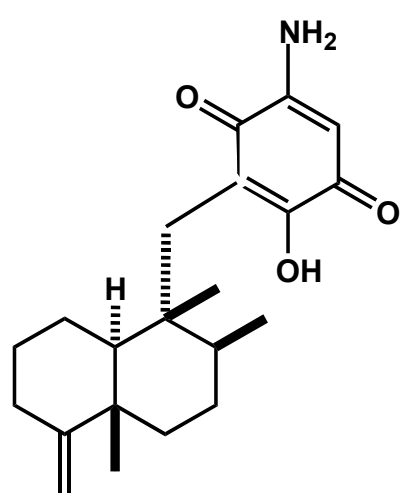

Figure 1. Chemical structure of smenospongine

\subsection{Effect of smenospongine on expression of 21 and phosphorylation of $R b$}

To demonstrate that the G1 phase arrest by smenospongine is attributed to p21 induction and inhibition of the phosphorylation of $\mathrm{Rb}$, which located in the downstream of p21 and is known to play a key role in cell cycle progression from $\mathrm{G} 1$ to $\mathrm{S}$ phase, $\mathrm{p} 21$ expression and phosphorylation of $\mathrm{Rb}$ in K562 cells were examined. A $15 \mu \mathrm{M}$ concentration of smenospongine was added to K562 cells. The cells treated for different length of time were collected, and the respective cell lysate was prepared for Western blotting analysis. The expression of p21 was detected after $12 \mathrm{~h}$ and increased in a timedependent manner until $48 \mathrm{~h}$ (Figure 3). Accordingly, the underphosphorylated Rb protein (URb) was also detected after $24 \mathrm{~h}$ and highly increased until $48 \mathrm{~h}$, indicating that the phosphorylation of $\mathrm{Rb}$ protein was inhibited by the smenospongine treatment for 24 to $48 \mathrm{~h}$. This result suggests that the p21$\mathrm{Rb}$ pathway might play an important role in the G1 phase arrest in K562 cells induced by smenospongine.

\subsection{Effect of smenospongine on activation of p21 promoter}

Since K562 cell is known as p53-negative cell [14], the p21 induction can be considered as p53independent. Furthermore, the induction of p21 mRNA expression by smenospongine was also observed by RT-PCR analysis (data not shown). This suggested the possibility that the $\mathrm{p} 21$ expression was induced by trans-activating p21 promoter. In order to investigate whether smenospongine can trans-activate p21 promoter or not, we transfected K562 cells with pWWP, a human wild-type p21 promoter-luciferase fusion plasmid. Luciferase assay was carried out with the transfected cells after DMSO, smenospongine or trichostatin A (TSA) treatment. TSA was used as a positive control, since it was previously reported to activate p21 promoter $[15,16]$. As a result, the luciferase activity of the smenospongine-treated K562 cells was not significantly increased, while the TSA-treated cells showed about 200 fold enhancement over those DMSO-treated, indicating that smenospongine can not activate p21 promoter (Figure 4). Therefore, we postulate that smenospongine might act by stabilizing p21 mRNA to increase mRNA and protein expression and lead to G1 arrest in K562 cells via p21-Rb pathway. The mechanism of smenospongine to induce apoptosis in HL60 and U937 cells, including effect on p21 expression, is under investigation. 
Figure 2. Effect of smenospongine on cell cycle distribution of K562, HL60, and U937 cells. A suspension of K562 (A), HL60 (B), and U937 (C) cells $\left(2 \times 10^{5}\right.$ cells $/ 2 \mathrm{ml} /$ well $)$ was incubated in the presence or absence of various concentrations of smenospongine in an 8 well plate under a $5 \% \mathrm{CO}_{2}$ atmosphere at $37^{\circ} \mathrm{C}$, respectively. After $24 \mathrm{~h}$, the cells were collected and dyed with DNA-Prep Reagents Kit for cell cycle analysis. The analysis was carried out with flow cytometer $(\lambda \mathrm{ex}=493 \mathrm{~nm}, \lambda \mathrm{em}=630 \mathrm{~nm})$ and quantified by ModFit Software.

A
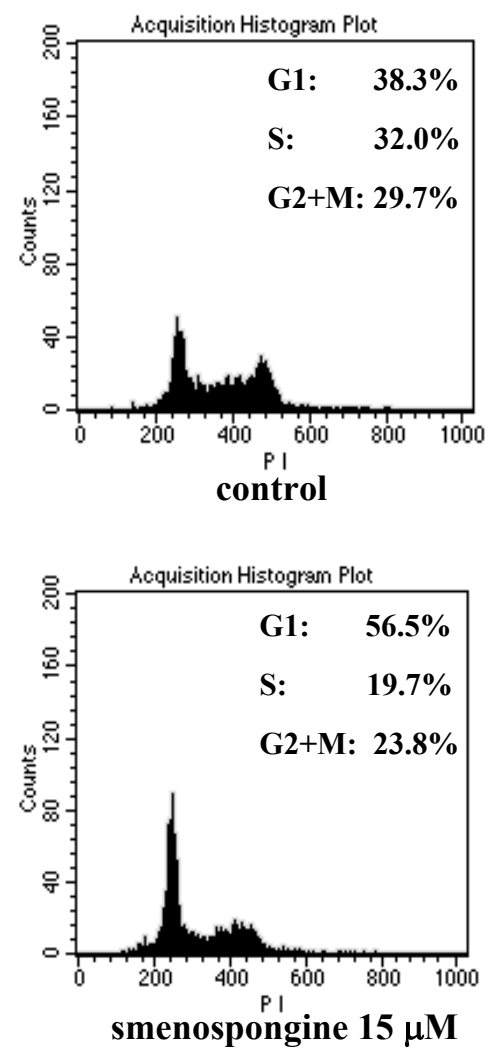

smenospongine $15 \mu \mathrm{M}$
B
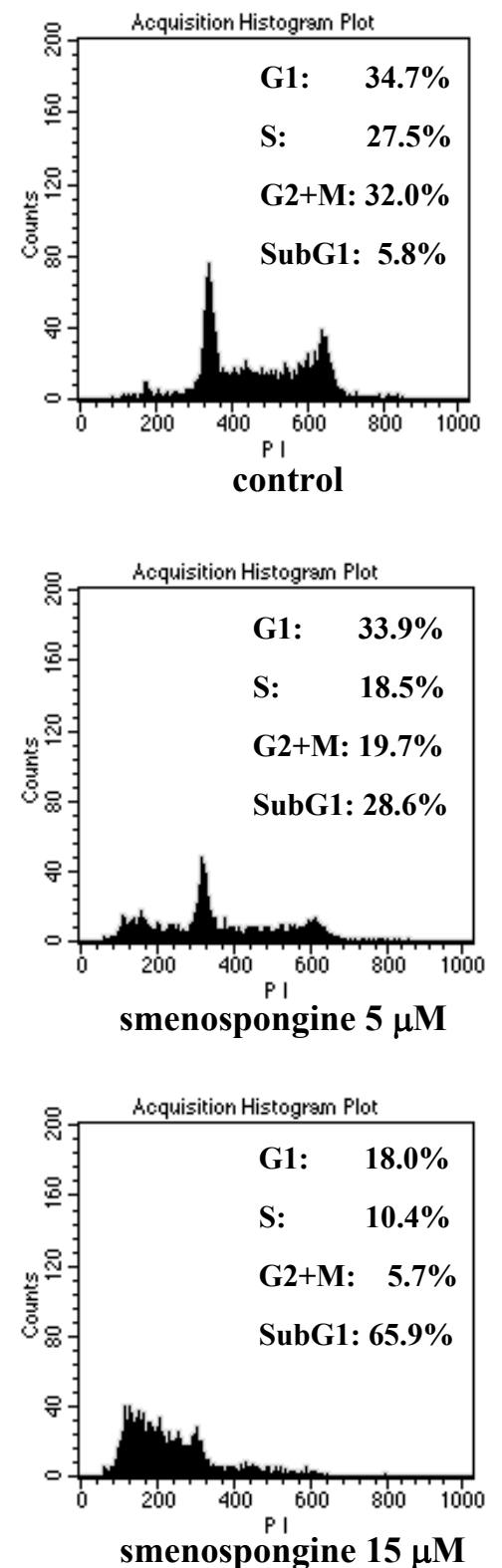

C
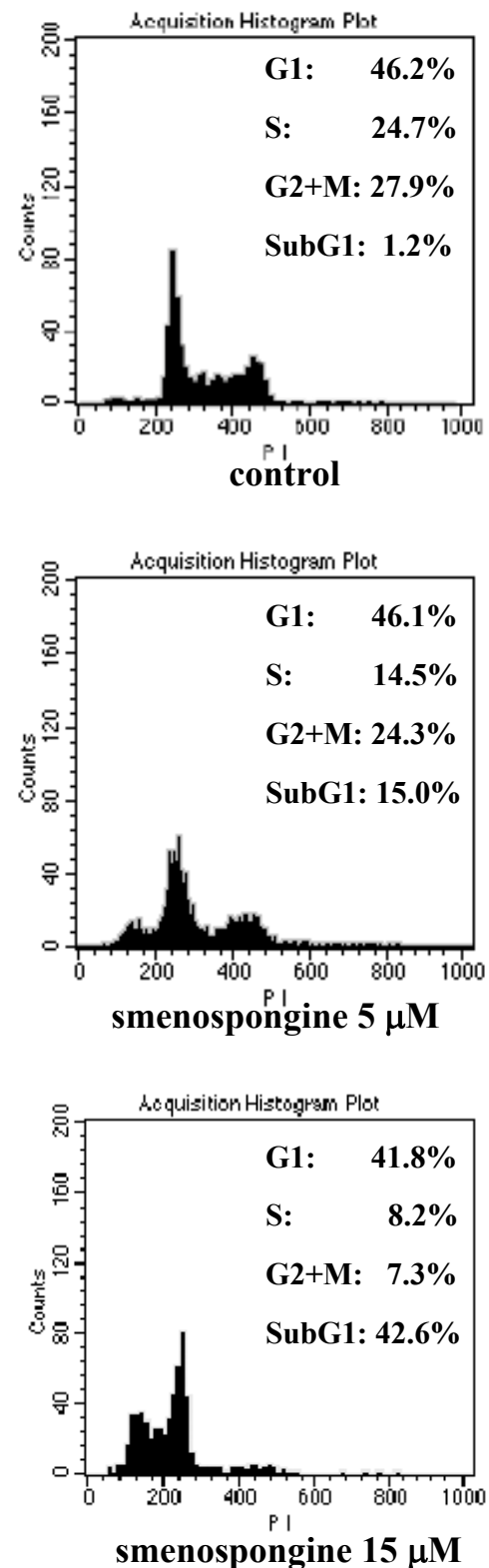
Figure 3. Effect of smenospongine on expression of p21 and phosphorylation of Rb. K562 cells $\left(1 \times 10^{6}\right.$ cells $\left./ 10 \mathrm{ml}\right)$ were incubated with $15 \mu \mathrm{M}$ smenospongine for the indicated time periods. The cell lysate was prepared and applied to 4-20\% SDS-PAGE. After being transferred to PVDF membrane, the blots were exposed to anti-Cip1/WAF-1/p21, antihuman underphosphorylated $\mathrm{Rb}(\mathrm{URb})$ or anti- $\beta$-actin antibody and then anti-mouse IgG HRP-conjugated antibody. The bound antibodies were visualized using an Enhanced Chemiluminescence (ECL) system.

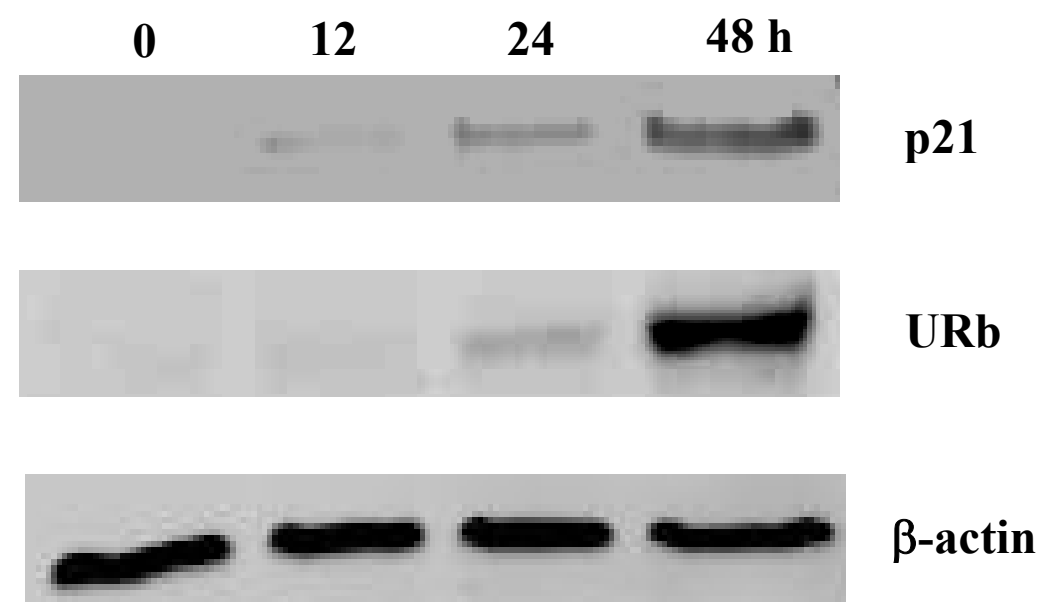

Figure 4. Activation of $\mathrm{p} 21$ promoter by smenospongine and TSA in the transfected K562 cells. K562 cells were transfected with pWWP, which is a human wild-type p21 promoterluciferase fusion plasmid, by DEAE-Dextran Transfection Kit. The cells were incubated at a density of $5 \times 10^{4}$ cells $/ \mathrm{ml}$ in a 12-well plate for $24 \mathrm{~h}$. $125 \mathrm{ng}$ of pWWP in DEAE-Dextran was added to perform transfection for $15 \mathrm{~min}$. The transfected cells were further incubated for $24 \mathrm{~h}$ and then treated by DMSO, smenospongine or TSA for another $24 \mathrm{~h}$. Finally, the cells were collected and the luminescence was measured by using MICRO LUMAT Plus LB96V. The activation of p21 promoter was evaluated by the relative light intensity compared with that of the control (cells treated with DMSO only).

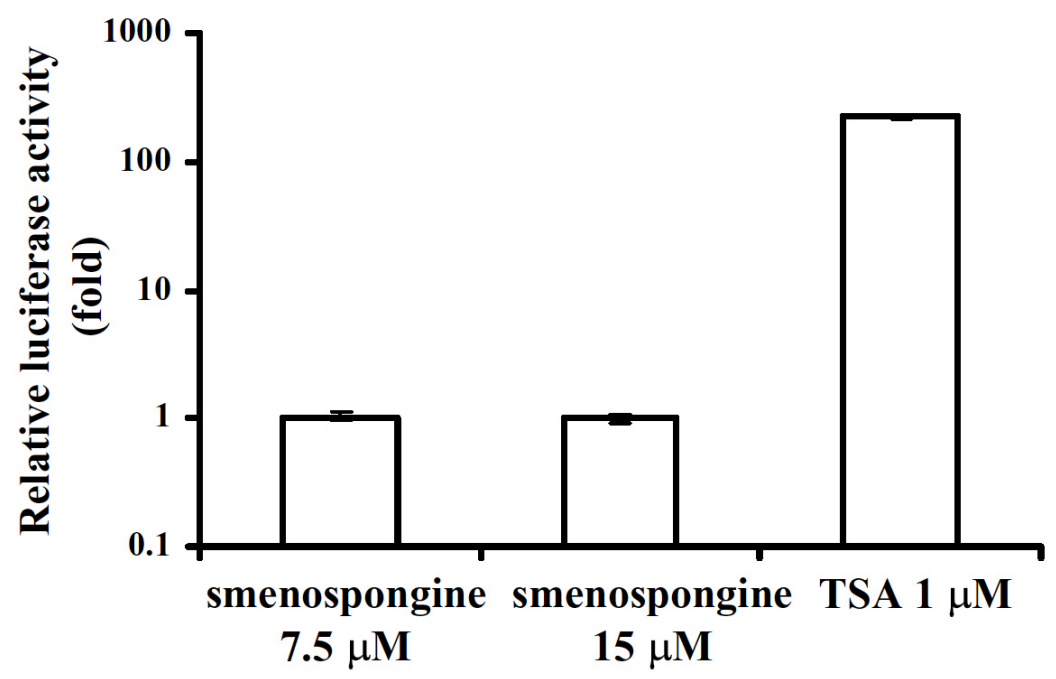




\section{Conclusions}

Smenospongine, a sesquiterpene aminoquinone isolated from the marine sponge of Dactylospongia elegans, induced G1 arrest in K562 cells, while induced apoptosis in HL60 and U937 cells. P21-Rb pathway is considered to play an important role in its effect of the G1 arrest in K562 cells.

\section{Experimental Section}

\subsection{Materials}

4-20\% gel cassette was purchased from Daiichi Pure Chemicals Co., Ltd. DNA-Prep Reagents Kit was from Coulter Co. RPMI 1640 and DMEM Medium were from Nissui Pharmaceutical Co., Ltd. Polyvinylidene fluoride (PVDF) membrane was from Amersham Pharmacia Biotec. UK Ltd. Other reagents were from Sigma Co., Ltd. or Wako Pure Chemical Industries Co., Ltd unless stated specifically.

\subsection{Cell culture}

K562, HL60, and U937 cells, which were provided by RIKEN Cell Bank, were routinely maintained in the RPMI 1640 (for K562 and U937) or DMEM (for HL60) medium supplemented with $10 \%$ fetal bovine serum, kanamycin $(100 \mu \mathrm{g} / \mathrm{mL})$ and glutamine $(0.44 \mathrm{mg} / \mathrm{mL})$ at $37^{\circ} \mathrm{C}$ in a humidified atmosphere containing $5 \% \mathrm{CO}_{2}$.

\subsection{Antibodies}

Anti-Cip1/WAF-1/p21 antibody was purchased from Upstate Biotechnology Inc.; anti-human underphosphorylated $\mathrm{Rb}$ antibody was from $\mathrm{BD}$ Biosciences; anti- $\beta$-actin antibody was from Sigma Co., Ltd; anti-mouse horseradish peroxidase (HRP) conjugated antibody was from Nacalai Tesque Inc.

\subsection{Isolation and identification of smenospongine}

Smenospongine was isolated from the marine sponge Dactylospongia elegans (collected in Indonesia in 2001) as described previously [12]. Briefly, the dried marine sponge was cut into pieces and extracted with methanol. The resulting methanol extract was subjected to solvent partition to give hexane, $90 \%$ methanol, $n-\mathrm{BuOH}$, and $\mathrm{H}_{2} \mathrm{O}$ soluble portions. The $90 \%$ methanol soluble portion was separated by repeated $\mathrm{SiO}_{2}$ column, reversed-phase column, and high-performance liquid chromatography (HPLC) to obtain smenospongine. The structure of smenospongine was identified by comparison of the mass and NMR data with those reported [17].

\subsection{Flow cytometric analysis of cell cycle}

The cell suspension $\left(2 \times 10^{5}\right.$ cells/2 ml/well) of K562, HL60 or U937 cells was placed in an 8-well plate and incubated for $24 \mathrm{~h}$ at $37^{\circ} \mathrm{C}$ under a $5 \% \mathrm{CO}_{2}$ atmosphere. The DMSO solution of the testing sample $(10 \mu \mathrm{l})$ was added and further incubated for $24 \mathrm{~h}$. The cells were harvested and collected, followed by staining with DNA-Prep Reagents Kit for $20 \mathrm{~min}$. Then, the supernatant was removed by 
centrifugation, and the cell pellets were re-suspended in $500 \mu \mathrm{l}$ of D-PBS (-). After being filtered with a 40- $\mu \mathrm{m}$ nylon mesh filter, the cell suspension was available for cell cycle analysis by flow cytometer $(\lambda \mathrm{ex}=493 \mathrm{~nm}, \lambda \mathrm{em}=630 \mathrm{~nm})$. The analysis result was quantified by ModFit software (Verity Software, Topsham, ME).

\subsection{Western blotting analysis}

The cell suspension $\left(1 \times 10^{6}\right.$ cells $\left./ 10 \mathrm{~mL}\right)$ of K562 cells was incubated in a flask together with $15 \mu \mathrm{M}$ of smenospongine for the indicated length of time under a $5 \% \mathrm{CO}_{2}$ atmosphere at $37^{\circ} \mathrm{C}$. The cells collected by centrifugation (1000 g for 3 min at $4^{\circ} \mathrm{C}$ ) were washed with cold PBS and treated with lysis buffer (50 mM Tris-HCl, pH7.2; 1\% NP-40; 0.25\% sodium deoxycholate; $150 \mathrm{mM} \mathrm{NaCl} ; 1 \mathrm{mM}$ EDTA; $1 \mathrm{mM}$ PMSF; $1 \%$ proteinase inhibitor cocktail) to furnish a cell lysate. Protein assay was carried out using a Bio-Rad protein assay kit. After boiling at $95^{\circ} \mathrm{C}$ for $5 \mathrm{~min}$ in the sample buffer (0.125 M Tris-HCl (pH 6.8), 10\% 2-mercaptoethanol, 4\% SDS, 10\% sucrose, 5\% bromophenol blue), the equal amounts of protein were subjected to SDS-Polyacrylamide gel electrophoresis (SDS-PAGE) and then transferred to PVDF membrane. The membrane was blocked with 5\% milk TBS (Tween PBS) and exposed to anti-Cip1/WAF-1/p21, anti-human underphosphorylated Rb, or anti- $\beta$-actin antibody and then anti-mouse IgG HRP-conjugated antibody. The bound antibodies were visualized using an Enhanced Chemiluminescence (ECL) system.

\subsection{Transfection of K562 cells and luciferase assay}

K562 cells were transfected with a human wild-type p21 promoter-luciferase fusion plasmid, pWWP, by DEAE-Dextran (CellPhect Transfection Kit, Amersham Pharmacia Biotech). The cells were incubated at a density of $5 \times 10^{4}$ cells $/ \mathrm{mL}$ in a 12-well plate for $24 \mathrm{~h}$. The $125 \mathrm{ng}$ of the reporter plasmid DNA, pWWP, in DEAE-Dextran was added to perform transfection for $15 \mathrm{~min}$. The transfected cells were further incubated for $24 \mathrm{~h}$ followed by DMSO, smenospongine or TSA treatment for another $24 \mathrm{~h}$. Finally, the cells were collected for luciferase assay, which was carried out by use of the luciferase assay system (E1501, Promega) as described previously [18]. The luminescence was measured by using MICRO LUMAT Plus LB96V (BERTHOLD) and WING LOW software. The activation of p21 promoter was evaluated by the relative light intensity compared with that of the control (cells treated with DMSO only) [18].

\section{Acknowledgements}

This study was supported by the Ministry of Education, Culture, Sports, Science, and Technology of Japan.

\section{References}

1. De Klein, A.; Van Kessel, A.G.; Grosveld, G.; Bartram, C.R.; Hagemeijer, A.; Bootsma, D.; Spurr, N.K.; Heisterkamp, N.; Groffen, J.; Stephenson, J.R. A cellular oncogene is translocated to the Philadelphia chromosome in chronic myelocytic leukaemia. Nature 1982, 300, 765-767. 
2. Lozzio, C.B.; Lozzio, B.B. Human chronic myelogenous leukemia cell-line with positive Philadelphia chromosome. Blood 1975, 45, 321-334.

3. Fausel, C. Targeted chronic myeloid leukemia therapy: seeking a cure. J. Manag. Care. Pharm. 2007, 13, 8-12.

4. Dan, S.; Naito, M.; Tsuruo, T. Selective induction of apoptosis in Philadelphia chromosomepositive chronic myelogenous leukemia cells by an inhibitor of BCR - ABL tyrosine kinase, CGP 57148. Cell Death Differ. 1998, 5, 710-715.

5. Melo, J.V.; Chuah, C. Resistance to imatinib mesylate in chronic myeloid leukaemia. Cancer Lett. 2007, 249, 121-132.

6. Nardi, V.; Azam, M.; Daley, G.Q. Mechanisms and implications of imatinib resistance mutations in BCR-ABL. Curr. Opin. Hematol. 2004, 11, 35-43.

7. Okabe, S.; Tauchi, T.; Nakajima, A.; Sashida, G.; Gotoh, A.; Broxmeyer, H.E.; Ohyashiki, J.H.; Ohyashiki, K. Depsipeptide (FK228) preferentially induces apoptosis in BCR/ABL-expressing cell lines and cells from patients with chronic myelogenous leukemia in blast crisis. Stem Cells Dev. 2007, 16, 503-514.

8. Byrd, J.C.; Marcucci, G.; Parthun, M.R.; Xiao, J.J.; Klisovic, R.B.; Moran, M.; Lin, T.S.; Liu, S.; Sklenar, A.R.; Davis, M.E.; Lucas, D.M.; Fischer, B.; Shank, R.; Tejaswi, S.L.; Binkley, P.; Wright, J.; Chan, K.K.; Grever, M.R. A phase 1 and pharmacodynamic study of depsipeptide (FK228) in chronic lymphocytic leukemia and acute myeloid leukemia. Blood 2005, 105, 959967.

9. Sasakawa, Y.; Naoe, Y.; Inoue, T.; Sasakawa, T.; Matsuo, M.; Manda, T.; Mutoh, S. Effects of FK228, a novel histone deacetylase inhibitor, on human lymphoma U-937 cells in vitro and in vivo. Biochem. Pharmacol. 2002, 64, 1079-1090.

10. Aoki, S.; Higuchi, K.; Isozumi, N.; Matsui, K.; Miyamoto, Y.; Itoh, N.; Tanaka, K.; Kobayashi, M. Differentiation in chronic myelogenous leukemia cell K562 by spongean sesterterpene. Biochem. Biophys. Res. Commun. 2001, 282, 426-431.

11. Aoki, S.; Kong, D.; Matsui, K.; Kobayashi, M. Erythroid differentiation in K562 chronic myelogenous cells induced by crambescidin 800, a pentacyclic guanidine alkaloid. Anticancer Res. 2004, 24, 2325-2330.

12. Aoki, S.; Kong, D.; Matsui, K.; Kobayashi, M. Smenospongine, a spongean sesquiterpene aminoquinone, induces erythroid differentiation in K562 cells. Anticancer Drugs 2004, 15, 363369.

13. Aoki, S.; Kong, D.; Matsui, K.; Rachmat, R.; Kobayashi, M. Sesquiterpene aminoquinones, from a marine sponge, induce erythroid differentiation in human chronic myelogenous leukemia, K562 cells. Chem. Pharm. Bull. (Tokyo) 2004, 52, 935-937.

14. Bi, S.; Hughes, T.; Bungey, J.; Chase, A.; De Fabritiis, P.; Goldman, J.M. p53 in chronic myeloid leukemia cell lines. Leukemia 1992, 6, 839-842.

15. Sowa, Y.; Orita, T.; Minamikawa, S.; Nakano, K.; Mizuno, T.; Nomura, H.; Sakai, T. Histone deacetylase inhibitor activates the WAF1/Cip1 gene promoter through the Sp1 sites. Biochem. Biophys. Res. Commun. 1997, 241, 142-150. 
16. Sowa, Y.; Orita, T.; Minamikawa-Hiranabe, S.; Mizuno, T.; Nomura, H.; Sakai, T. Sp3, but not $\mathrm{Sp} 1$, mediates the transcriptional activation of the p21/WAF1/Cip1 gene promoter by histone deacetylase inhibitor. Cancer Res. 1999, 59, 4266-4270.

17. Kondracki M. L., G.M. Smenospongine: a cytotoxic and antimicrobial aminoquinone isolated from smenospongia SP. Tetrahedron Lett. 1987, 28, 5815-5818.

18. Aoki, S.; Kong, D.; Suna, H.; Sowa, Y.; Sakai, T.; Setiawan, A.; Kobayashi, M. Aaptamine, a spongean alkaloid, activates p21 promoter in a p53-independent manner. Biochem. Biophys. Res. Commun. 2006, 342, 101-106.

Samples Availability: Not available.

(C) 2008 by the authors; licensee Molecular Diversity Preservation International, Basel, Switzerland. This article is an open-access article distributed under the terms and conditions of the Creative Commons Attribution license (http://creativecommons.org/licenses/by/3.0/). 